\title{
Một số hợp chất terpenoid phân lập từ cây lá diễn (Dicliptera chinensis (L.) Nees)
}

\author{
Vũ Đức Lợi ${ }^{1, *}$, Phạm Kim Thoa ${ }^{1}$, Bùi Thị Xuân ${ }^{1}$, \\ Nguyễn Hữu Tùng ${ }^{1}$, Nguyễn Thành $\mathrm{Nam}^{2}$, \\ Trịnh Nam Trung ${ }^{2}$, Nguyễn Tiến Vững ${ }^{3}$ \\ ${ }^{1}$ Khoa Y Duoơc, Đại học Quốc gia Hà Nọi, \\ 144 Xuân Thủy, Cầu Giấy, Hà Nội, Việt Nam \\ ${ }^{2}$ Học viện Quân y, số 160 Phùng Hưng, Hà Đông, Hà Nội, Việt Nam \\ ${ }^{3}$ Viện Pháp y Quốc gia, số 41 Nguyễn Đình Chiểu, Hai Bà Trung, Hà Nội, Việt Nam \\ Nhận ngày 3 tháng 4 năm 2017 \\ Chỉnh sửa ngày 21 tháng 4 năm 2017; Chấp nhận đăng ngày 14 tháng 6 năm 2017
}

\begin{abstract}
Tóm tắt: Từ dịch chiết ethanol của lá cây lá diễn (Dicliptera chinensis (L.) Nees) thu hái ở tỉnh Nam Định, sử dụng phương pháp sắc ký đã phân lập được hai hợp chất terpenoid. Các hợp chất

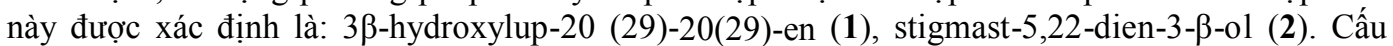
trúc của các hợp chất này dựa trên các dữ liệu phổ khối lượng và cộng hưởng từ hạt nhân kết hợp so sánh với dữ liệu phổ được công bố trong tài liệu tham khảo. Đây là 2 hợp chất lần đầu tiên được phân lập từ lá cây lá diễn.
\end{abstract}

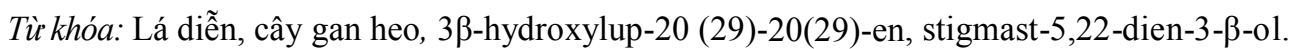

\section{1. Đặt vấn đề}

Cây lá diễn hay còn gọi là cây Gan heo (Dicliptera chinensis (L.) Nees), họ Ô rôAcanthaceae đã được biết đến là cây được dùng làm thuốc, thực phẩm $[1,2]$. Cây này mọc hoang, hay mọc ở những chố ẩm ướt. Cây lá diễn được ứng dụng trong khá nhiều bài thuốc dân gian, đem lại hiệu quả chữa bệnh cao như dùng để chữa: mắt đỏ sưng đau, viêm họng sưng đau sốt cao, lên sởi, viêm phổi nhẹ, viêm ruột thừa cấp, viêm gan cấp, viêm ruột, cách chữa bệnh chỉ cần lấy vài lá tươi rửa sạch giã nát xoa đắp hoặc lấy dịch lá nhỏ vào chỗ sưng

\footnotetext{
* Tác giả liên hệ. ĐT.: 84-989313325.

Email: ducloi82@gmail.com

https://doi.org/10.25073/2588-1132/vnumps.4052
}

đau hoặc dùng toàn cây khô hoặc tươi sắc uống [1]. Ở nước ngoài, cây này đã có một số nghiên cứu về tác dụng chống oxy hóa và chống viêm $[6,8]$. Tại Việt Nam, cây này có mọc tại nhiều tỉnh ở khu vực phía Bắc nước ta. Cây dễ trồng và thời gian cho thu hoạch nhanh (khoảng 3 tháng). Cây này hoàn toàn có thể sử dụng làm nguyên liệu làm thuốc, nếu được nghiên cứu đầy đủ về thành phần hóa học, tác dụng sinh học. Vì vậy, nhóm nghiên cứu đã chiết xuất, phân lập một số thành phần và công bố trong bài báo này.

\section{2. Đối tượng và phương pháp nghiên cứu}

\section{1. Đối tương nghiên cúu}


Mẫu cây lá diễn được thu hái vào tháng 6 năm 2016 tại huyện Trực Ninh, tỉnh Nam Định. Mẫu thực vật đã được Viện Sinh thái và Tài nguyên sinh vật giám định tên khoa học là: Dicliptera chinensis (L.) Nees., họ Ồ rôAcanthaceae, mẫu được lưu giữ tại Khoa $\mathrm{Y}$ Dược, ĐHQGHN.

\subsection{Dung môi, hóa chất}

Các dung môi dùng trong chiết xuất, phân lập như ethanol (EtOH), n-hexan, ethyl acetat (EtOAc), dicloromethan (DCM)... đều đạt tiêu chuẩn công nghiệp và được chưng cất lại trước khi dùng. Dung môi phân tích gồm $\mathrm{MeOH}$, nhexan, EtOAc, $\mathrm{H}_{2} \mathrm{O}$ dùng để phân tích sắc ký đều đạt tiêu chuẩn phân tích. Pha tĩnh dùng trong sắc ký cột là silica gel pha thường $(0,040$ - 0,063 mm, Nicalai Tesque Inc., Nhật Bản), YMC ODS-A $(50 \mu \mathrm{m}$, YMC Co. Ltd., Nhật Bản). Bản mỏng tráng sẵn trên đế nhôm loại pha thường Kieselgel $60 \mathrm{~F}_{254}$ và pha đảo TLC Silica gel 60 RP-18 $\mathrm{F}_{254 \mathrm{~S}}$ (Merck, Damstadt, Đức). Phát hiện chất bằng đèn tử ngoại ở hai bước sóng $254 \mathrm{~nm}$ và $365 \mathrm{~nm}$ hoặc dùng thuốc thử là dung dịch $\mathrm{H}_{2} \mathrm{SO}_{4} 10 \%$ hơ nóng để phát hiện vết chất.

\subsection{Thiết bị, dụng cu}

- Sắc ký cột: sắc ký cột sử dụng silicagel cõ̃ hat $0,063-0,200 \mathrm{~mm}$ (Merck) và cỡ hat 0,040 $0,063 \mathrm{~mm}$ (Merck) với các loại cột sắc ký có kích cỡ khác nhau.

- Phổ cộng hưởng tù hạt nhân: NMR được ghi trên máy Bruker Avance $500 \mathrm{MHz}$ tại Viện Hóa học, Viện Hàn lâm Khoa học và Công nghệ Việt Nam.

- Phổ khối ESI-MS: đo trên máy AGILENT 1260 Series LC-MS ion Trap (Agilent Technologies, Hoa Kỳ)

- Nhiệt độ nóng chảy: đo trên máy SMP10 BioCote, Khoa Y Dược, ĐHQGHN.

- Góc quay cưc riêng: đo trên máy PLR-4, MRC scientific instruments, Khoa Y Dược, ĐHQGHN.

\subsection{Chiết tách và phân lập chất}

Mẫu lá cây lá diễn được phơi khô, nghiền thành bột $(6,5 \mathrm{~kg})$, sau đó chiết trong ethanol $\left(3 \times 8\right.$ lít) bằng thiết bị chiết siêu âm $\left(50^{\circ} \mathrm{C}, 3\right.$ h). Dịch chiết được lọc qua giấy lọc, gộp dịch chiết lại và cất loại dung môi ở áp suất giảm thu được 630,0 g cặn chiết ethanol. Lấy $120 \mathrm{~g}$ cặn chiết này phân tán vào 1,2 lít nước cất và tiến hành chiết phân bố lần lượt với $n$-hexan và ethylacetat (mỗi loại $3 \times 1,5$ lít). Các dịch chiết $n$ - hexan, ethylacetat được cất thu hồi dung môi thu được các cặn dịch tương ứng $n$ - hexan $(\mathrm{H}$, $31,0 \mathrm{~g})$ và $(\mathrm{E}, 56,0 \mathrm{~g})$ và lớp nước $(\mathrm{N}, 33,0 \mathrm{~g})$.

Cắn n-hexan $(30,0 \mathrm{~g})$ được phân lập trên cột sắc ký với chất hấp phụ silicagel, sử dụng hệ dung môi n-hexan: axeton (25/1). Hứng dịch rửa giải vào các ống nghiệm và kiểm tra bằng SKLM, gộp các ống nghiệm giống nhau lại và bay hơi dung môi thu được 3 phân đoạn H1 $(9,4 \mathrm{~g}), \mathrm{H} 2(8,6 \mathrm{~g}), \mathrm{H} 3(6,5 \mathrm{~g})$. Tiến hành sắc ký cột cắn phân đoạn $\mathrm{H} 2$ với chất hấp phụ silicagel, hệ dung môi rửa giải n-hexan: ethyl acetat $(10 / 1,5 / 1,2,5 / 1)$, thu được 3 phân đoạn nhỏ H2.1 (2,3g), H2.2 (2,5g), H2.3 (3,0g). Phân đoạn H2.1 phân tách trên cột silicagel pha thường với hệ dung môi rửa giải diclometan:metanol (10/1) thu được hợp chất 1 (55mg). Phân đoạn H2.2 phân tách trên cột silicagel pha thường với hệ dung môi rửa giải $n$-hexan: ethylacetat $(2 / 1)$ thu được chất sạch 2 (62 mg).

\section{Kết quả và bàn luận}

Hơp chất 1: 33-hydroxylup-20 (29)-20(29)-en

Tính chất: là một chất kết tinh ở dạng tinh thể màu trắng, nhiệt độ nóng chảy: $188-189^{\circ} \mathrm{C}$, không tan trong nước, tan trong ether, ethanol, methanol.

Phổ khối lượng ESI-MS: $m / z \quad 427$ $[\mathrm{M}+\mathrm{H}]^{+}$. Phổ NMR trình bày ở Bảng 1 .

Phổ khối lượng ESI-MS xuất hiện pic ion phân tử tại $m / z 427[\mathrm{M}+\mathrm{H}]^{+}$cho phép xác định công thức phân tử là $\mathrm{C}_{30} \mathrm{H}_{50} \mathrm{O}(\mathrm{M}=426)$. Phổ NMR chỉ ra rằng hợp chất này là hợp chất triterpenoid có khung lup-20(29)-en. 
Bảng 1. Dữ liệu phổ NMR của hợp chất $\mathbf{1}$ và chất so sánh $\mathbf{L}$

\begin{tabular}{|c|c|c|c|c|c|}
\hline Vị trí C & DEPT & $\delta_{\mathbf{C}}(\mathbf{L}) \mathrm{ppm}$ & $\delta_{\mathbf{C}}$ (1) $\mathrm{ppm}$ & $\delta \mathbf{H}(\mathbf{1}) \mathrm{ppm}, J: \mathrm{Hz}$ & $\delta_{\mathbf{H}}$ (L) ppm, J: Hz \\
\hline 1 & $\mathrm{CH}_{2}$ & 38,7 & 38,8 & & \\
\hline 2 & $\mathrm{CH}_{2}$ & 27,5 & 27,4 & & \\
\hline 3 & $\mathrm{CH}$ & 79,0 & 79,1 & $3,17 \mathrm{dd}(11,5 ; 5,0)$ & $3,18 \mathrm{dd}(11,5 ; 5,0)$ \\
\hline 4 & $\mathrm{C}$ & 38,9 & 38,9 & & \\
\hline 5 & $\mathrm{CH}$ & 55,3 & 55,4 & & \\
\hline 6 & $\mathrm{CH}_{2}$ & 18,3 & 18,4 & & \\
\hline 7 & $\mathrm{CH}_{2}$ & 34,3 & 34,4 & & \\
\hline 8 & $\mathrm{C}^{2}$ & 40,9 & 40,8 & & \\
\hline 9 & $\mathrm{CH}$ & 50,5 & 50,5 & & \\
\hline 10 & $\mathrm{C}$ & 37,2 & 37,2 & & \\
\hline 11 & $\mathrm{CH}_{2}$ & 21,0 & 20,9 & & \\
\hline 12 & $\mathrm{CH}_{2}$ & 25,2 & 25,2 & & \\
\hline 13 & $\mathrm{CH}$ & 38,1 & 38,1 & & \\
\hline 14 & $\mathrm{C}$ & 42,9 & 42,8 & & \\
\hline 15 & $\mathrm{CH}_{2}$ & 27,5 & 27,4 & & \\
\hline 16 & $\mathrm{CH}_{2}$ & 35,6 & 35,6 & & \\
\hline 17 & $\mathrm{C}^{2}$ & 43,0 & 43,1 & & \\
\hline 18 & $\mathrm{CH}$ & 48,0 & 48,4 & & \\
\hline 19 & $\mathrm{CH}$ & 48,3 & 48,1 & $2,38 \mathrm{ddd}(11,0 ; 11,0 ; 6,0)$ & $2,36 \mathrm{ddd}(11,0 ; 11,0 ; 6,0)$ \\
\hline 20 & $\mathrm{C}$ & 150,9 & 150,9 & & \\
\hline 21 & $\mathrm{CH}_{2}$ & 29,9 & 29,9 & & \\
\hline 22 & $\mathrm{CH}_{2}$ & 40,0 & 40,1 & & \\
\hline 23 & $\mathrm{CH}_{3}$ & 28,0 & 28,0 & $0,98 \mathrm{~s}$ & $0,96 \mathrm{~s}$ \\
\hline 24 & $\mathrm{CH}_{3}$ & 15,3 & 15,4 & $0,76 \mathrm{~s}$ & $0,76 \mathrm{~s}$ \\
\hline 25 & $\mathrm{CH}_{3}$ & 16,1 & 16,1 & $0,85 \mathrm{~s}$ & $0,84 \mathrm{~s}$ \\
\hline 26 & $\mathrm{CH}_{3}$ & 16,0 & 16,1 & $1,05 \mathrm{~s}$ & $1,04 \mathrm{~s}$ \\
\hline 27 & $\mathrm{CH}_{3}$ & 14,6 & 14,5 & $0,94 \mathrm{~s}$ & $0,95 \mathrm{~s}$ \\
\hline 28 & $\mathrm{CH}_{3}^{3}$ & 18,0 & 18,0 & $0,79 \mathrm{~s}$ & $0,79 \mathrm{~s}$ \\
\hline 29 & $\mathrm{CH}_{3}^{3}$ & 19,3 & 19,3 & $1,67 \mathrm{~s}$ & $1,69 \mathrm{~s}$ \\
\hline 30 & $\mathrm{CH}_{2}$ & 109,3 & 109,4 & $4,68 \mathrm{~s}$ & $4,66 \mathrm{~s}$ \\
\hline
\end{tabular}

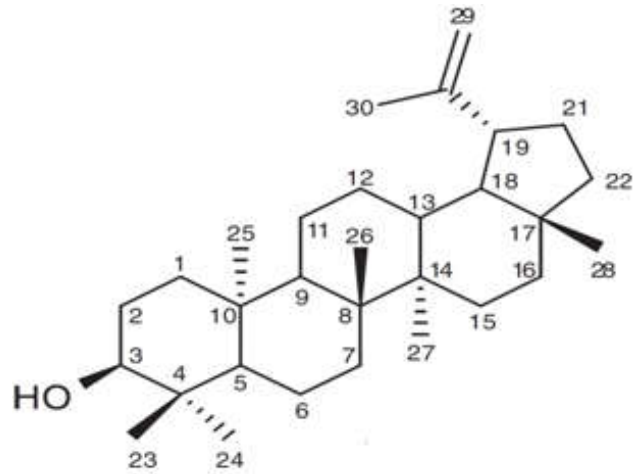

Hình 1. Cấu trúc của hợp chất $\mathbf{1}$. 
Phổ ${ }^{1}$ H-NMR cho thấy tín hiệu của 7 nhóm methyl $\mathrm{CH}_{3}$ cộng hưởng trong vùng trường mạnh, trong đó có 6 nhóm methyl bậc ba $\mathrm{CH}_{3}$ tại các giá trị $\delta_{\mathrm{H}} 0,98(3 \mathrm{H}, \mathrm{s}, \mathrm{H}-23)$; $0,76(3 \mathrm{H}, \mathrm{s}, \mathrm{H}-24) ; 0,85$ (3H, s, H-25); 1,05 $(3 \mathrm{H}, \mathrm{s}, \mathrm{H}-26) ; 0,94(3 \mathrm{H}, \mathrm{s}, \mathrm{H}-27) ; 0,79(3 \mathrm{H}, \mathrm{s}$, $\mathrm{H}-28)$ và 1 tín hiệu $\mathrm{CH}_{3}$ tại $1,67(3 \mathrm{H}$, br $\mathrm{s}, \mathrm{H}-$ 29) thuộc nhánh isopropenyl. Tín hiệu tại 3,38 $(1 \mathrm{H}, \mathrm{dd}, J=11,5 ; 5,0 \mathrm{~Hz}, \mathrm{H}-3)$ khẳng định sự có mặt của một nhóm oximethin $(\mathrm{CH}-\mathrm{O})$. Tín hiệu 2 proton thuộc nhánh isopropenyl tại $\delta_{\mathrm{H}} 4,68\left(1 \mathrm{H}, \mathrm{d}, J=1,0 \mathrm{~Hz}, \mathrm{H}_{\mathrm{a}}-30\right)$ và $4,56(1 \mathrm{H}$, $\mathrm{d}, J=1,0 \mathrm{~Hz}, \mathrm{Hb}^{-30)}$.

Phổ ${ }^{13} \mathrm{C}$-NMR của 1 bao gồm tín hiệu của 30 nguyên tử carbon. Phân tích các tín hiệu trên phổ DEPT xác nhận được gồm 7 carbon methyl $\left(\mathrm{CH}_{3}\right), 11$ carbon methylen $\left(\mathrm{CH}_{2}\right), 6$ carbon methin $(\mathrm{CH})$ và 6 carbon bậc bốn $(\mathrm{C})$. Nhánh isopropenyl được xác nhận bởi các tín hiệu của 1 carbon methylen tại 109,4 (C-29) cùng với tín hiệu của 1 carbon bậc bốn tại 150,9 (C-20) và 1 carbon methyl 19,3 (C-29). Ngoài ra, tín hiệu của 1 carbon oximethin tại 79,1 (C-3) cho phép xác nhận có 1 nhóm hydroxyl trong phân tử của hợp chất.

So sánh số liệu phổ NMR của hợp chất 1 với số liệu của hợp chất triterpen khung lup20(29)-en đã biết là 3ß-hydroxylup-20(29)-en $[3,4,9]$, nhận thấy có sự giống nhau ở các vị trí tương ứng (Bảng 1). Nhận định này được khẳng định thêm khi xem xét đến hằng số tương tác $J$ của proton $\mathrm{H}-3$. Proton $\mathrm{H}-3$ cộng hưởng tại 3,17 (dd, $J=11,5 ; 5,0 \mathrm{~Hz}$ ), tín hiệu doublet này cho thấy hằng số tương tác $J$ lớn vì vậy $\mathrm{H}$ 3 ở vị trí axial $(a)$ hay alpha dẫn tới $\mathrm{OH}$ tại $\mathrm{C}-3$ là equatorial $(e)$. Từ các phân tích nêu trên hợp chất 1 được xác định là 3ß-hydroxylup-20 (29)20(29)-en

Hợp chất 2: Stigmast-5,22-dien-3- $\beta$-ol

Tinh thể hình kim, không màu, không mùi.

${ }^{1} \mathrm{H}-\mathrm{NMR}\left(400 \mathrm{MHz}, \mathrm{CDCl}_{3}\right), \delta(\mathrm{ppm})$ : $5,35(1 \mathrm{H}, \mathrm{d}, J=5 \mathrm{~Hz}, \mathrm{H}-6), 5,11(1 \mathrm{H}, \mathrm{dd})$ $5,03(1 \mathrm{H}, \mathrm{dd}), 3,52(1 \mathrm{H}, \mathrm{m}, \mathrm{H}-3), 1,01(3 \mathrm{H}, \mathrm{s}$, $\mathrm{H}-19)$.

${ }^{13} \mathrm{C}-\mathrm{NMR}\left(100 \mathrm{MHz}, \mathrm{CDCl}_{3}\right), \delta$ (ppm): 140,6 (C-5), 138,5 (C-22), 129,2 (C-23), 121,8 (C-6), 71,9 (C-3), 56,8 (C-14), 55,9 (C-17),
51,3 (C-9), 50,2 (C-24), 42,3 (C-4), 42,2 (C12), 40,4 (C-20), 37,2 (C-7), 36,3 (C-1), 36,1 (C-13), 33,9 (C-25), 31,8 (C-8), 31,8 (C-13), 31,6 (C-16), 29,1 (C-2), 28,2 (C-28), 26,0 (C-15), 24,2 (C-11), 21,3 (C-26), 21,0 (C-21), 19,4 (C27), 18,7 (C-19), 12,2 (C-29), 12,0 (C-18).

Hợp chất 2 thu được dưới dạng tinh thể hình kim, không màu, không mùi, nhiệt độ nóng chảy $155-157^{\circ} \mathrm{C},[\alpha]_{\mathrm{D}}^{25}=-45^{\circ}(\mathrm{c}=0,05$ trong $\mathrm{CHCl}_{3}$ ).

Trên phổ ${ }^{1} \mathrm{H}-\mathrm{NMR}$ và ${ }^{13} \mathrm{C}-\mathrm{NMR}$ cho thấy có ba proton olefinic ở $\delta 5,35(1 \mathrm{H}, \mathrm{d}, J=$ 5,0 Hz, H-6), 5,11 (1H, dd, H-22), 5,03 $(1 \mathrm{H}$, dd, H-23) ứng với ba cacbon ở $\delta 121,8$ (C-6), 138,5 (C-22), 129,2 (C-23). Dựa vào dữ kiện lý hóa và so sánh phổ của stigmast-5,22-dien3 - $\beta$-ol đã công bố $[5,7]$ thấy có sự trùng khớp vì vậy hợp chất $\mathbf{2}$ được xác định là stigmast5,22-dien-3- $\beta$-ol.

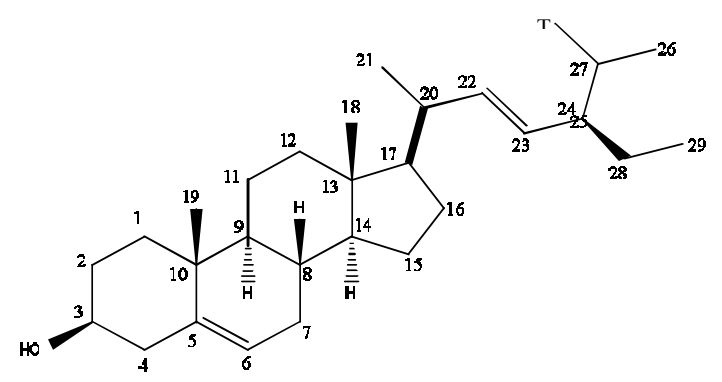

Hình 2. Cấu trúc hóa học của hợp chất 2.

\section{Kết luận}

Đã sử dụng phương pháp ngâm chiết với dung môi $\mathrm{EtOH} 90 \%$ và bằng phương pháp sắc ký cột phân lập được 2 hợp chất từ phần lá của cây lá diễn thu hái tại huyện Trực Ninh, tỉnh Nam Định. Cấu trúc các hợp chất này được xác định thông qua kết quả đo nhiệt độ nóng chảy, góc quay cực riêng, phổ tử ngoại- khả kiến, phổ khối, phổ cộng hưởng hạt nhân và so sánh với các dữ liệu công bố của các hợp chất liên quan. Hai hợp chất được xác định là $3 \beta$-hydroxylup20 (29)-20(29)-en (1) và stigmast-5,22-dien-3$\beta$-ol (2). Đây là lần đầu tiên 2 hợp chất này được phân lập từ cây lá diễn (Dicliptera chinensis (L.) Nees). 


\section{Lời cảm ơn}

Nghiên cứu này được tài trợ bởi Đại học Quốc Gia Hà Nội, đề tài "Nghiên cứu phát triển nguồn dược liệu chuẩn và nguyên liệu làm thuốc nhỏ mắt từ cây thuốc thuộc chi Dicliptera", mã số: QG.17.28.

\title{
Terpenenoid Compounds Isolated from the Leaf of Dicliptera Chinensis (L.) Nees.
}

Vu Duc Loi ${ }^{1}$, Pham Kim Thoa ${ }^{1}$, Bui Thi Xuan ${ }^{1}$, Nguyen Huu Tung ${ }^{1}$, Nguyen Thanh Nam ${ }^{2}$, Trinh Nam Trung ${ }^{2}$, Nguyen Tien Vung ${ }^{3}$

${ }^{I}$ School of Medicine and Pharmacy, Vietnam National University, Hanoi, 144 Xuan Thuy, Cau Giay District, Hanoi, Vietnam

${ }^{2}$ Vietnam Militery Medical University, 160 Phung Hung, Ha Dong District, Hanoi, Vietnam

${ }^{3}$ National Institute of Forensic Medicine, 41 Nguyen Dinh Chieu, Hai Ba Trung District, Hanoi, Vietnam

\begin{abstract}
From theethanol extract of the leaf of Dicliptera chinensis (L.) Nees. collected in Nam Dinh province, two compounds (1-2) were isolated by chromatographic methods. These compounds

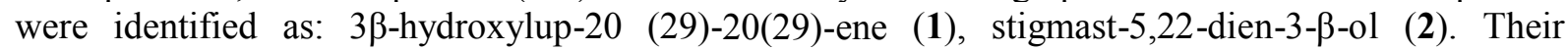
structures were elucidated by spectroscopic methods, including MS and NMR. These compounds were isolated from the leaf of Dicliptera chinensis (L.) Nees for the first time..
\end{abstract}

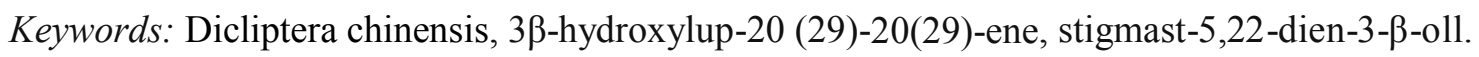

